\title{
Trapping of $\Sigma^{+}$hyperons in nuclei
}

\author{
Sławomir Wycech ${ }^{1, \star}$ and Kristian Piscicchia ${ }^{2,3}$ \\ ${ }^{1}$ National Centre for Nuclear Studies, Hoża 69, 00-681 Warsaw, Poland \\ ${ }^{2}$ LNF-INFN, Italy \\ ${ }^{3}$ Museo Storico della Fisica e Centro Studi e Ricerche Enrico Fermi
}

\begin{abstract}
The nuclear capture of $K^{-}$studied by FINUDA discovered a puzzling low momentum component in the spectrum of final $\Sigma^{+}$hyperon. This component is interpreted here as the effect of Gamov state formed by the hyperon. Such state is quasi-localized in space with a radius in between the hyper-nuclear and the hyper-atomic radius. Experimental and theoretical consequences of this discovery are studied.
\end{abstract}

\section{Introduction}

The formation of the hyperon and meson pairs $\Sigma^{ \pm}, \pi^{\mp}$ following the $K^{-} p$ capture in nuclei has been studied by a number of experimental techniques. Early experiments performed in nuclear emulsion and $\mathrm{He}(\mathrm{D})$ bubble chambers [1] concentrated on studies of total $P_{\Sigma \pi}$ momenta or invariant masses $M_{\Sigma \pi}$. These experiments allow to test invariant mass of the $K^{-} p$ pair in the subthreshold region. The purpose of such research has been to learn the structure of $\Lambda(1405)$ resonance located below the $K^{-} p$ threshold. Properties of the latter may be detected with a simultaneous measurement of : $M_{\Sigma, \pi}, P_{\Sigma \pi}$ and the ratio of two formation rates $\sigma\left(\Sigma^{+}, \pi^{-}\right) / \sigma\left(\Sigma^{+}, \pi^{-}\right)=R_{ \pm}\left(M_{\Sigma, \pi}\right)$. This ratio depends strongly on the invariant mass and reflects an interference of the resonant amplitude with a background one. Recent experiments by FINUDA collaboration allowed fairly precise measurements of both, the meson and the hyperon momenta [2]. The reaction in question is

$$
K^{-6} L i \rightarrow \Sigma^{ \pm} \pi^{\mp} R
$$

with some residual system $R$, apparently $R \approx^{5} \mathrm{He}$. In agreement with old measurements it has been found that the spectrum of $\Sigma^{-}$momentum is described by a regular peak centered at $\sim 200 \mathrm{MeV} / \mathrm{c}$. On the other hand, the spectrum of $\Sigma^{+}$momentum indicates two peaks apparently due to two different formation mechanisms. The additional low energy peak is located in the segment $<0,50>\mathrm{MeV} / \mathrm{c}$ corresponds to centroid kinetic energy of $\sim 0.3 \mathrm{MeV}$, very small on the nuclear scale.

The difference in spectra is apparently related to different hyperon charge which matters at low momenta. The $R_{ \pm}\left(M_{\Sigma, \pi}\right)$ is also dramatically changed for those events which involve low energy hyperons. This finding has an indirect precedence. The European $K^{-}$collaboration studied subthreshold ratio $R_{ \pm}$in light emulsion nuclei. Results obtained by Keane [3] indicated an anomaly of $R_{ \pm}$around $M_{\Sigma, \pi} \sim 1395 \mathrm{MeV}$ some $35 \mathrm{MeV}$ below the $K^{-} p$ threshold. This region of $M_{\Sigma, \pi}$ corresponds essentially to $p(\Sigma) \sim 0$ events. The anomaly itself was studied in analysis [4] motivated by the idea of

\footnotetext{
^e-mail: wycech@fuw.edu.pl
} 
Dalitz [5] that in addition to the baryonic component a sizable fraction of $\Lambda(1405)$ may be due to a direct three quark component. Now, in the light of the FINUDA finding this anomaly may be related to the low momentum peak in the $\Sigma^{+}$spectrum.

Here, the low momentum peak is attributed to the $\Sigma^{+}$hyperon trapping in the residual nucleus. A fraction of hyperons is trapped into a Gamov state formed by the attractive nuclear potential and repulsive Coulomb barrier. In section 2 we discuss properties of such $\left(\Sigma^{+},{ }^{5} \mathrm{He}\right)$ state formed in an intermediate state of $K^{-}$capture in ${ }^{6} \mathrm{Li}$. We conclude that the Gamov state may offer explanation of the low momentum peak. However, a good quantitative description requires more data. Next, a new chapter in the physics of $\Sigma$-nucleus interactions ma be opened.

\section{The origin of anomalous threshold peak in the $\Sigma^{+}$momentum}

The mechanism for the peak formation is sequential : (1) the $K$ meson undergoes capture $K^{-} p \rightarrow$ $\Sigma^{+} \pi^{-}$. Residual nucleus $R$ is a spectator (2) the $\Sigma^{+}$hyperon and $R$ are trapped by Coulomb barrier into Gamov state (3) the Gamov state decays into low momentum $\Sigma^{+}$and $R$.

If the Gamov state is very large the internal momenta are small and $\Sigma^{+}$is released with a negligible momentum. In such conditions, the final meson momentum $p_{\pi}$ is $\sim 180 \mathrm{MeV} / \mathrm{c}$ and the momentum taken by the hyperon is well fixed at value $p_{\Sigma} \sim p_{\pi} m_{\Sigma} /\left(m_{\Sigma}+m_{R}\right) \sim 20 \mathrm{MeV}$ which is the center of the experimental peak in the $\mathrm{Li}$ case.

The Gamov state of $\Sigma^{+}$hyperon is a quasi-localised state formed in the continuum by an attractive nuclear potential and repulsive Coulomb barrier. Historically, such states were introduced to describe the $\alpha$ decay of heavy nuclei. Quantum mechanics allows to understand the unstable state of $\alpha$ particle localised within a nucleus and decaying via sub-barrier transition. The technical description requires solution of the corresponding Schroedinger equation with the outgoing wave boundary condition. Such solutions are possible for negative energies and describe bound states. For positive energies such solutions might also exists but with complex energies and imaginary parts of these energies give lifetimes of such systems. There is a difference between Gamov states of $\alpha$ formed in heavy nuclei and $\Sigma^{+}$formed in light nuclei. In the latter case Coulomb barriers are low, energies of the Gamow states are low and the hyperon has a sizable probability to stay outside the nucleus. Moreover, the hyperon is absorbed by nuclei and the wave equation has to be determined in complex domain anyway, with the help of a complex optical potential. Here we use $V_{n}=\left[V_{o}-i W_{o}\right] \rho(r)$ and search for $S$ wave quasi-bound states of positive energies

$$
-\frac{1}{2 M} \frac{d R}{d^{2} r}+\left(V_{\text {culomb }}+V_{n}\right) R=\left(E_{G}-i \Gamma / 2\right) R .
$$

The three step mechanism specified above generates a product of three factors in the formula for $\mathrm{K}^{-}$ capture amplitude

$$
A_{\Sigma}=C_{G} D\left(p_{\pi}\right) \widetilde{\Phi}(q) .
$$

The first factor $G$ is an amplitude ( here it is an unspecified parameter ) for the $\Sigma^{+}$to be captured into Gamov state. Propagation of the Gamov hyper-nuclear system is described by

$$
D\left(p_{\pi}\right)=\left[E_{\Sigma}\left(p_{\Sigma}\right)+E_{R}\left(p_{R}\right)-E_{G}\left(p_{\pi}\right)+i \Gamma_{G} / 2\right]^{-1}
$$

where $E_{\Sigma}, E_{R}$ and $E_{G}$ are the hyperon residual nucleus and Gamov-system energies. As we are in the continuum there is a pole in $D$. The last factor $\widetilde{\Phi}$ is the Fourier transform of the wave function $R / r$ expressed in terms of relative momentum $\left.\left.\mathbf{q}=\mathbf{p}_{r} m_{\Sigma} /\left(m_{\Sigma}+m_{R}\right)\right)-\mathbf{p}_{\Sigma} m_{R} /\left(m_{\Sigma}+m_{R}\right)\right)$ Profiles of the final $p(\Sigma)$ distribution presented below are given by the last two factors, both are important. 
Several values of well depth $V_{o}$ have been tried to study the effect of nucleus on the Gamov state. Apparently there exist no $\Sigma^{+}$hypernuclei ( other than ${ }_{\Sigma}^{4} \mathrm{He}$ [6]) and we use potentials which do not generate bound states. The mere existence of a Gamov state puts strong limitations on $V_{o}$ from above as well as from below. The limiting values in ${ }^{5} \mathrm{He}$ are in the bracket ( -28 till $-26 \mathrm{MeV}$ ) depending on absorptive strength tried in the region $W_{o}<10 \mathrm{MeV}$ motivated by the hyperhelium width. Differential equation (2) is integrated numerically to distances of $50 \mathrm{fm}$ For technical simplicity the Coulomb potential is suppressed at distances exceeding $28 \mathrm{fm}$. These boundary conditions are good enough to obtain stability of the method and a three digit precision of energies and widths. As a consistency check we use the condition $\int \operatorname{Im} V_{n}|\Phi|^{2}=2 \Gamma$. The complex energy $E_{G}-i \Gamma / 2$ is obtained with " asymptotic" wave function $(R / r)^{2}$ which drops to the level of $10^{-8}$ of the initial one. Under the cut-off condition one can define mean square radii of the Gamov systems. For the cases presented in the figure these are less than $15 \mathrm{fm}$.
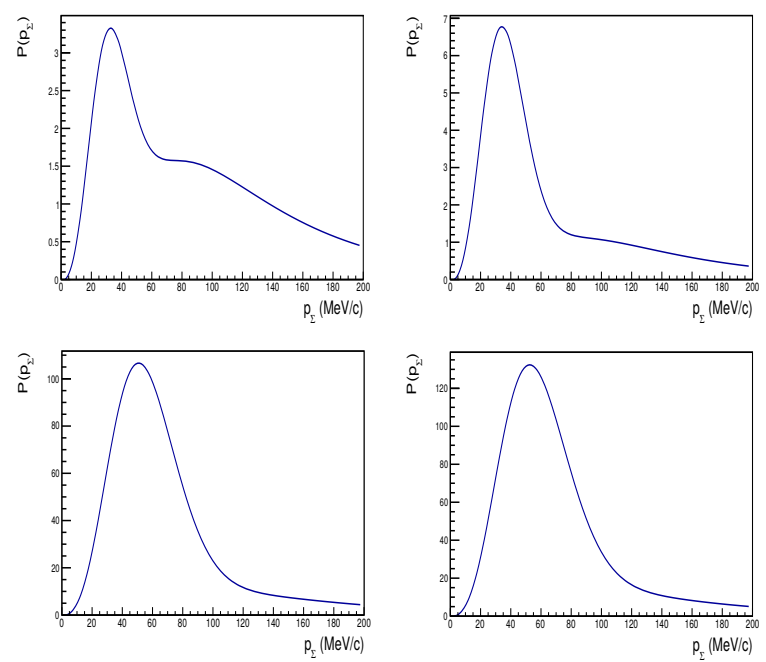

Figure 1. Calculated profiles of the low energy peaks in $\Sigma^{+}$momentum distribution. Corresponding quantum numbers of Gamov states $\left(E_{G}, \Gamma\right)$ in $\mathrm{MeV}$ are: up left $(0.068,1.35)$, up right $(0.03,1.38)$, down left $(0.12,0.19)$ down right $(0.02,0)$

Amplitude $|A|^{2}$ averaged over final 3-body phase space generates the shape of low energy momentum peak. Several examples are plotted in the figure. The results indicate existence of threshold peak centered around 50 but not around $20 \mathrm{MeV} / \mathrm{c}$ found by FINUDA. There might be several effects which are missing: (1) The residual nucleus ( ${ }^{5} \mathrm{He}$ is unstable with $\Gamma \sim 0.5 \mathrm{MeV}$ ), its density is calculated [7] but not tested. (2) There may be some momentum dependence of the Gamov state formation amplitude $G$. (3) Some loss of the final hyperon momentum in the target may take place. These questions may be answered with more data and those are forthcoming [8].

An additional asymmetry in the $\Sigma^{ \pm}, \pi^{\mp}$ formation may also stem from $\Sigma, N$ threshold enhancement [9]. This effect we find to be weak and also unlikely to produce a narrow peak. 


\section{Conclusions}

The description of the anomalous low energy momentum distribution $P\left(p_{\Sigma}\right)$ in terms of the Gamov state is in a qualitative consistency with the FINUDA data. The mere fact of existence of the low energy peak puts strong limitation on the hyperon nucleus potential. The shape of of the peak may help to determine the strength of $\Sigma$ absorption in nuclei. These two pieces of information are to some extent equivalent to a detection of ( apparently nonexistent ) $\Sigma$ hypernuclei. The FINUDA data is related to a hyperon ${ }^{5} \mathrm{He}$ system. The only, so far, detected sigma hypernucleus has also been found in He. It would be very interesting to check the existence of Gamov states of $\Sigma^{+}$also in heavier nuclei. Positive answer would open a new branch of hypernuclear spectroscopy.

The full understanding of $p_{\Sigma}$ spectrum in particular the weight of the anomalous peak relative to the standard spectrum might be very informative, perhaps more than the $\Sigma$ hypernuclear spectroscopy would be.

\section{References}

[1] E.H.S. Burhop, High Energy Phys. Vol. 3 Acad. Press. N. York, (1967)

[2] M. Angelo for FINUDA, Phys. Lett. B 704, 474 (2011)

[3] D.F. Keane, Thesis, Univ. College Belfield, Dublin, (1981)

[4] L.R. Staronski and S. Wycech, J. Phys. G 13, 1361 (1987)

[5] R.H. Dalitz and J.G. McGinley, Low and Intermediate Energy KN Physics ed. E. Ferrari and G. Violini, Springer, 1980, p. 381

[6] T. Nagae, et al., Phys. Rev. Lett. 80, 1608 (1998)

[7] T. Neff and H. Feldmeier, Nucl. Phys. A. 738, 357 (2004)

[8] AMADEUS/KLOE project: $K^{-}$capture in $\mathrm{He}, \mathrm{B}, \mathrm{C}, \mathrm{Al}$, private information

[9] H. Machner et al., Nucl. Phys. A 901, 65 (2013) 